\title{
Behavioural activation $v$. antidepressant medication for treating depression in Iran: randomised trial
}

\author{
Latif Moradveisi, Marcus J. H. Huibers, Fritz Renner, Modabber Arasteh and Arnoud Arntz
}

\section{Background}

Behavioural activation might be a viable alternative to antidepressant medication for major depressive disorder.

\section{Aims \\ To compare the effectiveness of behavioural activation and treatment as usual (TAU, antidepressant medication) for major depressive disorder in routine clinical practice in Iran.}

\section{Method}

Patients with major depressive disorder $(n=100)$ were randomised to 16 sessions of behavioural activation $(n=50)$ or antidepressant medication ( $n=50)$ (IRCT138807192573N1). The main outcome was depression, measured with the Beck Depression Inventory (BDI) and the Hamilton Rating Scale for Depression (HRSD), assessed at 0, 4, 13 and 49 weeks.

\section{Results}

Symptom reduction was greater in the behavioural activation group than in the TAU group on both the BDI and the HRSD at 13 and 49 weeks in multilevel analysis. Baseline depression severity was a moderator, with relatively better effects for behavioural activation in individuals who were more severely depressed. Also, there was better retention in the behavioural activation than in the TAU group.

\section{Conclusions}

Behavioural activation is a viable and effective treatment for people with major depressive disorder, especially for those who are more severely depressed, and it can successfully be disseminated into routine practice settings in a non-Western country such as Iran.

\section{Declaration of interest}

None.
Major depressive disorder is a common mental health problem ${ }^{1}$ and there are many studies that have demonstrated the effectiveness of antidepressant medication in treating it. $^{2-5}$ Thus, antidepressants have become the standard treatment for depression, ${ }^{6,7}$ despite their limitations such as problems with side-effects, refusal by patients to take them and considerable relapse after discontinuation. Psychological treatments might offer a viable alternative. ${ }^{8-10}$ In non-Western countries, the use of antidepressant medication is even more common, due to the limited availability of psychotherapy. This means there is a need for better dissemination of relatively simple but effective psychological treatments. Behavioural activation is such a candidate, given its effectiveness and relatively simple protocol.

Behavioural activation is based on the behavioural component of cognitive-behavioural therapy (CBT) for treating depression. ${ }^{11}$ A study by Jacobson $e t$ al $^{12}$ found that behavioural activation was as effective as a full CBT package for treating depression. In a subsequent trial by the same research group, Dimidjian et al compared behavioural activation with antidepressant medication and CBT. ${ }^{13}$ They found that behavioural activation was as effective as antidepressant medication, and even outperformed CBT, especially in individuals who were more severely depressed. Moreover, participants originally treated with antidepressant medication and later a pill placebo experienced more relapse at the 2-year follow-up than individuals treated with behavioural activation or $\mathrm{CBT}^{8}{ }^{8}$ Thus, for prevention of recurrence, behavioural activation and CBT were superior to medication discontinuation. Finally, more recently, two meta-analyses unanimously found that behavioural activation interventions are as effective as CBT. ${ }^{14,15}$ One study by Ekers et al ${ }^{16}$ compared behavioural activation (delivered by nurse practitioners) with treatment as usual (TAU) in a routine clinical setting. The behavioural activation was superior to TAU and the authors concluded that it might be easily delivered by mental health professionals.
The present study was set up to document the effectiveness of behavioural activation when implemented in clinical practice in Iran after a short period of training. We reasoned that there is a need for psychological treatment for depression in Iran as an alternative to antidepressant medication, which although readably available is not very popular due to its association with mental illness (creating stigma) and because of side effects. From the effective psychological treatments available, behavioural activation seemed the most easy to implement. Training and treatment were based on the published behavioural activation protocol, ${ }^{17,18}$ and none of the developers of behavioural activation or specialists were involved. Thus, the study's aim was to investigate whether a simple psychological treatment, behavioural activation, would be a viable alternative to antidepressant medication for people with depression, when implemented in a non-Western country. We compared behavioural activation to antidepressant medication (TAU) in Iran including drop-out rates, effectiveness in reducing depressive symptoms, response and remission rates, and relapse at approximately 1 year. We also assessed whether initial depression severity would moderate condition differences.

\section{Method}

\section{Participants}

Participants included 100 people with depression between the ages of 18 and 70 years, selected from 174 referrals. Inclusion criteria were: a primary diagnosis of major depressive disorder according to the DSM-IV-TR, ${ }^{19}$ confirmed by the Structured Clinical Interview for the DSM-IV-TR (SCID-CT) ${ }^{20}$ a score of $\geqslant 19$ on the Beck Depression Inventory (BDI-II $)^{21}$ and $\geqslant 14$ on the 17-item Hamilton Rating Scale for Depression (HRSD); ${ }^{22}$ and written consent to participate in the study. Exclusion criteria were: a lifetime diagnosis of bipolar disorder or psychosis; organic brain 
syndrome; intellectual disability; substantial and imminent suicide risk; a current (within the past 6 months) diagnosis of alcohol or drug misuse or dependence, or a positive toxicology screen; a primary diagnosis other than major depressive disorder; unfavourable antidepressant medication response within the preceding year; unstable medical condition; medication use that would complicate antidepressant administration; known allergy to antidepressant medication/sertraline; pregnancy or a plan to become pregnant; and inability to read and understand the study's instruments.

Participants were recruited through the media and poster advertisements $(n=45,45 \%)$, word of mouth $(n=8,8 \%)$, and referral from other mental health clinics $(n=28,28 \%)$ and general practitioners $(n=19,19 \%)$. Participants diagnosed with major depressive disorder through telephone screening were referred to the mental health clinic for further assessment. Psychiatrists confirmed diagnoses and checked the eligibility of participants who completed the assessment. When eligibility was confirmed, participants were randomised by an independent coordinator using a computer-generated list based on blocks of four, created by an independent statistician at Kurdistan University of Medical Science. Fifty participants were randomly assigned to each condition (IRCT138807192573N1). Baseline HRSD was used as a severity index. Participants were allocated to therapists and psychiatrists based on their availability.

The study was powered to detect a medium to large effect between conditions, with $80 \%$ power and a 0.05 level of significance. To compensate for participants dropping out, the original $n=80$ on which the power analysis was based was extended to $n=100$. The study was approved by the local medical ethics committee of Kurdistan University of Medical Science. Participants were treated at Ghods Psychiatric Hospital and the Mental Health Clinic in Sanandaj, Kurdistan Province. The study was conducted from November 2009 to September 2011.

\section{Therapists}

Behavioural activation was conducted by two counsellor psychologists and the first author (L.M.); they had 6 years' clinical experience on average. Training in behavioural activation was provided by L.M., a master-level psychologist, in $20 \mathrm{~h}$ over 2 weeks. Therapists participated in an on-site consultation meeting held almost twice a week, led by L.M. Four psychiatrists provided TAU (antidepressant medication); all were certified with an average of approximately 5 years of clinical experience. Training and supervision was provided by M.A.

\section{Treatments}

\section{Behavioural activation}

The behavioural activation model we used was based on the two behavioural activation manuals by Martell et al.$^{17,18}$ Behavioural activation interventions are behaviour-based and specific cognitive interventions are prohibited. The focus is on the participant's behaviours and the environmental context in which the behaviours take place; acting according to goals, not to feelings; and using an activity chart to schedule people's activities and follow the relationship between activity and mood. Identifying secondary problems such as avoidance patterns and depressive ruminations are important because they play a role in maintaining depression. Most individuals with depression withdraw from social activities, thereby minimising distress in the short term but creating long-term difficulties. Behavioural activation tries to break down the pattern of avoidance and utilises behavioural techniques to target depressive ruminations.
Behavioural activation therapists deal with ruminating as a behaviour rather than engaging with or challenging the contents of ruminative thoughts.

Participants received 16 sessions over 12 weeks (as opposed to the earlier trials by Jacobson $e a^{12}$ and Dimidjian et $a l^{13}$ in which patients received 24 sessions over 4 months). For the first 4 weeks there were two sessions per week, and for the following 8 weeks there was one session per week.

\section{Treatment as usual (antidepressant medication)}

For TAU, we chose sertraline, a selective serotonin reuptake inhibitor (SSRI), the major reason being the availability of it in Iran. The participants in the TAU group were not offered any psychotherapeutic interventions by their psychiatrists but they established treatment rapport to support the continuation of treatment. For the first 4 weeks of the treatment, participants received one session per week and for the next 8 weeks they received one session every 2 weeks. For the first session, psychiatrists saw participants for $20 \mathrm{~min}$ and the following sessions lasted approximately $10 \mathrm{~min}$ to monitor pharmacotherapy and side-effects. The maximum dosage of sertraline was $100 \mathrm{mg}$ per day. In week 1 , participants in the TAU group received $25 \mathrm{mg} /$ daily of sertraline, the dosage in week 2 increased to $50 \mathrm{mg}, 75 \mathrm{mg}$ in week 4, and $100 \mathrm{mg}$ in week 6 up to week 12 . Psychiatrists could reduce the dosage temporarily in the case of side-effects and then increase the dosage to the previous level. The maximum dosage was $100 \mathrm{mg}$ because as a rule, higher dosages do not increase antidepressant efficacy, but may increase the risk of adverse effects. ${ }^{23}$ All decisions about the dosage were made by the supervising psychiatrist (M.A). After 12 weeks, it was up to participants whether they continued or stopped antidepressant medication. In the case of continuation, participants had to pay for their medication.

\section{Measures}

\section{Diagnostic measures (baseline)}

We used SCID-I, ${ }^{20}$ which is a semi-structured clinical interview, to evaluate DSM-IV-TR Axis I diagnoses. We used SCID-II ${ }^{24}$ for diagnosing personality disorders (effects are reported separately).

\section{Outcome measures}

The modified 17-item HRSD and the 21-item BDI-II, both assessing depressive symptoms, were outcome measures. The HRSD was taken by evaluators who were masked to group. Assessments were conducted at baseline, and at 4, 13 and 49 weeks. In accordance with the Dimidjian et al study, ${ }^{13}$ each session was preceded by the HRSD (TAU group) and the BDI-II (behavioural activation group), administered by assistants who were masked to group; for participants who dropped out of treatment we used these data as the last observation in the analysis (see Statistical analysis).

\section{Baseline severity}

The baseline HRSD was used as the depression severity measure.

\section{Response, remission and relapse criteria}

Response indicates substantial symptomatic improvement, whereas remission means that symptoms lie within the normal range. Response was defined as at least a 50\% reduction from baseline on both the HRSD and BDI-II. Remission was defined as scores of $\leqslant 7$ on the HRSD and $\leqslant 10$ on the BDI. Relapse 
was defined as no longer meeting the remission criterion at 49 weeks in patients who remitted at 13 weeks.

\section{Statistical analysis}

Multilevel analysis was used to estimate change in depression severity over time. We applied intention-to-treat analysis, with the HRSD and BDI as dependent variables, by including all available scores. Visual inspection suggested linear and quadratic time effects; we therefore modelled time linearly and quadratically. We used an unstructured covariance structure for repeated measures, with time, time squared and condition as fixed effects. We computed effect sizes for continuous outcomes (Cohen's $d$ and $r$ ) from the multilevel estimates (Cohen's $d=$ (Baseline mean mean at time $i) /(\sqrt{ }$ baseline variance $)$ for within-condition change; $d=$ (difference between means of condition at time $i$ )/ $\sqrt{ }($ residual variance at time $i)$ for differences between conditions; $r=\sqrt{ }(F /(F+$ d.f. $))$. For participants who dropped out of treatment, we used the last observation with the associated time, and estimated missing HRSD scores (for those in the behavioural activation group) from changes on the BDI, and missing BDI scores (for those in the TAU group) from changes on the HRSD, using regression-derived equations (1 BDI unit $=1.3 \mathrm{HRSD}$ unit).
All treatment drop-out took place before the mid-treatment assessment. We repeated the analyses without these estimates as a sensitivity analysis.

For the test of moderation by baseline symptom severity, we entered time, time squared, the standardised baseline severity score and condition as covariates in the model. There was only one baseline assessment and therefore we included HRSD and BDI scores at weeks 4,13 and 49 as dependent variables in the analyses. The baseline severity $x$ condition and the time $x$ baseline severity $\times$ treatment condition interactions are of primary interest in this analysis. In all models, treatment condition was centred at $-1 / 2$ and $1 / 2$. Analyses were conducted using SPSS19 for Windows.

\section{Results}

\section{Participant enrolment}

Figure 1 shows the flow of participants. Of 174 participants originally recruited, 74 did not meet selection criteria: 20 did not meet criteria for major depressive disorder, 9 had too low severity scores on the HRSD or BDI-II, 37 had other primary diagnoses/problems (generalised anxiety disorder $(n=10)$, medical problems $(n=6)$, post-traumatic stress disorder $(n=1)$, bipolar

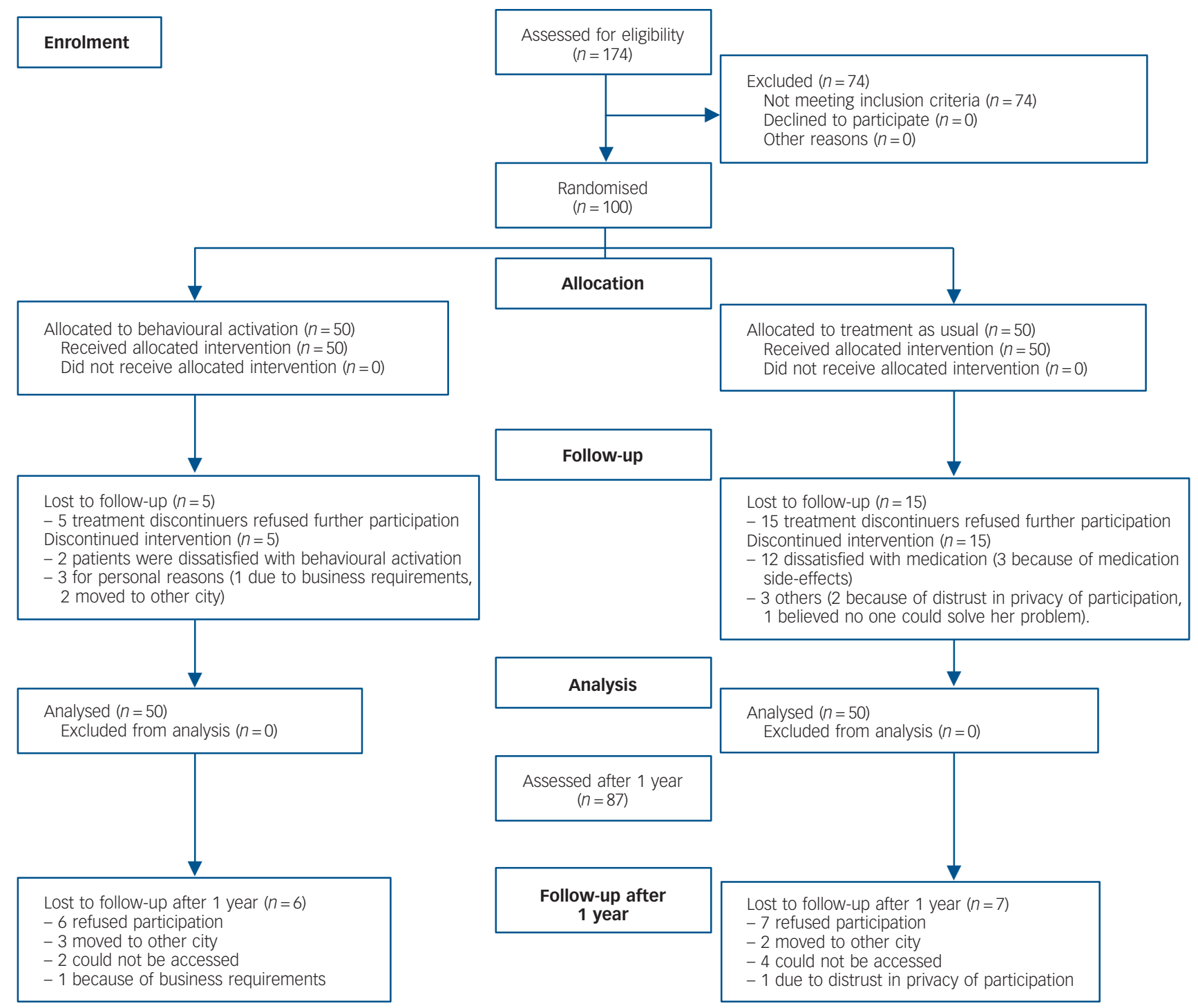

Fig. 1 CONSORT 2010 flow diagram. 
disorder $(n=5)$, substance dependence $(n=3)$, obsessive-compulsive disorder $(n=8)$, positive alcohol screen $(n=3)$, opiate addiction for more than 4 years $(n=1)$ ), antidepressant use unsuccessfully for more than 2 years $(n=5)$, and 3 were pregnant. The remaining 100 were randomised.

\section{Baseline}

Table 1 presents baseline sample demographic and clinical characteristics. Treatment groups were not significantly different on any of the variables.

\section{Drop out}

Five participants (10\%) dropped out of the behavioural activation group, as opposed to 15 participants (30\%) from the TAU group. In the behavioural activation group, two people were dissatisfied with behavioural activation and three had personal reasons for dropping out (one due to the participant's business; two moved to another city). In the TAU group, 12 participants were dissatisfied with the medication (3 due to medication side-effects), 2 distrusted the privacy of participation, and 1 believed no one could solve their problem. The difference in drop-out rate was significant $\left(\chi_{(1, n=100)}^{2}=6.25, P=0.012\right.$, odds ratio $(\mathrm{OR})=3.86$, $95 \%$ CI $1.28-11.64)$.

\section{Change in depression severity over time}

Tables 2 and 3 summarise the multilevel analyses results. In the primary analyses (Table 2), there were significant interactions between time and condition (HRSD $r=0.29$; BDI $r=0.25$ ) and between time squared and condition (HRSD $r=0.29$; BDI $r=0.22$ ). Figure 2 shows the stronger symptom reduction over time on the HRSD and BDI in the behavioural activation group at 13 weeks.

Within-condition changes (all $P<0.001$; Cohen's $d$ s with baseline s.d.) are presented in Table 4 . Response to behavioural activation differed from TAU at 13 weeks (mean HRSD-change difference 3.09, 95\% CI 1.71-4.47, $d=0.88, P<0.001$; mean BDI-change difference $3.3495 \%$ CI $1.63-5.05, d=0.76$, $P<0.001$ ) and at 49 weeks (mean HRSD-change difference 2.34, 95\% CI 0.84-3.84, $d=0.61, P<0.001$; mean BDI-change difference 3.67, 95\% CI 2.10-5.23, $d=0.92, P<0.001$ ); statistics based on residual variances at 13 and 49 weeks respectively.
Sensitivity analyses yielded comparable results (Table 2). We also tested whether referral type influenced the results by adding referral (self-referral $v$. professional-referral) as a covariate (online Table DS1 and Fig. DS1). The table reports results after deleting non-significant higher order effects involving referral. In short, referral did not change the condition $\times$ time and condition $\times$ time squared effects, but professionally referred patients had a steeper decrease in depressive symptoms in the behavioural activation group than self-referred patients, with no (HRSD) or the opposite effect (BDI) in the TAU group.

\section{Moderation by baseline severity}

The three-way interactions between time (respectively time squared), condition and baseline severity were not significant; linear time-effect: $F(1,75.85)=1.49, P=0.17$ for the HRSD, and $F(1,97.20)=1.03, P=0.31$ for the BDI; quadratic time-effect: $F(1,75.85)=1.49, P=0.23$ for the HRSD and $F(1,94.80)=0.96$, $P=0.33$ for the BDI (results not shown in Table 3). After removing the three-way interactions from the model, there were significant interactions between treatment condition and baseline severity (Table 3 ), indicating that baseline severity moderated treatment outcome at 4,13 and 49 weeks. As is shown in Fig. 3, participants with higher baseline severity had relatively lower HRSD and BDI scores in the behavioural activation group than in the TAU group.

\section{Remission and response}

Among study completers, remission and response rates for the behavioural activation group at 13 weeks were 41 out of 45 (91.1\%) and 44 out of $45(97.8 \%)$ participants. For the TAU group, these rates were 24 out of $35(68.6 \%)$ and 33 out of 35 (94.3\%). Behavioural activation differed significantly from TAU in remission rate $\left(\chi_{(1, n=80)}^{2}=6.93, P<0.01, \mathrm{OR}=4.69,95 \% \mathrm{CI}\right.$ $1.35-16.40)$; but not in response rate $\left(\chi_{(1, n=80)}^{2}=0.67, P=0.42\right.$, $\mathrm{OR}=2.66,95 \%$ CI $0.23-30.67)$.

\section{Remission, response and relapse at 49 weeks}

A total of 87 participants were available for assessment at 49 weeks. Remission and response rates for the behavioural activation group were 29 out of $44(65.9 \%)$ and 39 out of $44(88.6 \%)$

\begin{tabular}{|c|c|c|c|c|c|c|c|}
\hline \multirow[b]{2}{*}{ Baseline characteristics } & \multirow{2}{*}{$\begin{array}{l}\text { Full sample } \\
\qquad(n=100)\end{array}$} & \multirow{2}{*}{$\begin{array}{l}\text { Medication } \\
\text { treatment } \\
(n=50)\end{array}$} & \multirow{2}{*}{$\begin{array}{l}\text { Behavioural } \\
\text { activation } \\
(n=50)\end{array}$} & \multicolumn{3}{|c|}{ Test statistic } & \multirow{2}{*}{$\begin{array}{c}P \\
\text { (2-tailed }\end{array}$} \\
\hline & & & & $\chi^{2}$ (d.f.) & $t$ (d.f.) & $U$ & \\
\hline Female, $n(\%)$ & $85(85.0)$ & $40(80.0)$ & $45(90.0)$ & $1.96(1)$ & & & 0.16 \\
\hline Age, years: mean (s.d.) & $31.37(8.97)$ & $32.62(10.17)$ & $30.12(7.47)$ & & $1.4(98)$ & & 0.16 \\
\hline Currently married or cohabiting, $n(\%)$ & $48(48.0)$ & $25(50.0)$ & $23(46.0)$ & $0.04(1)$ & & & 0.84 \\
\hline College graduate, $n$ (\%) & $40(40.0)$ & $19(38.0)$ & $21(42.0)$ & $0.37(1)$ & & & 0.54 \\
\hline College student, $n$ (\%) & $23(23.0)$ & $10(20.0)$ & $13(26.0)$ & $2.68(1)$ & & & 0.10 \\
\hline Employed outside home, $n$ (\%) & $35(35.0)$ & $19(38.0)$ & $16(32.0)$ & $0.38(1)$ & & & 0.58 \\
\hline Current episode length, months: mean (s.d.) & $5.87(2.14)$ & $5.69(1.97)$ & $6.06(2.31)$ & & $1.01(98)$ & & 0.31 \\
\hline \multicolumn{8}{|l|}{ Severity } \\
\hline HRSD overall, mean (s.d.) & $21.37(5.32)$ & $21.62(542)$ & $21.12(5.26)$ & & $-0.47(98)$ & & 0.64 \\
\hline Number of prior episodes, mean (s.d.) & $1.03(0.80)$ & $1.00(0.72)$ & $1.06(0.88)$ & & & 1222.5 & 0.63 \\
\hline Recurrent depression, $n$ (\%) & $73(73.0)$ & $38(76.0)$ & $35(70.0)$ & $0.45(1)$ & & & 0.49 \\
\hline Prior admissions to psychiatric hospital, $n$ (\%) & $2(2.0)$ & 0 & $2(4.0)$ & & & & $0.49^{\mathrm{a}}$ \\
\hline Any current Axis I diagnosis, $n$ (\%) & $11(11.0)$ & $4(8.0)$ & $7(14.0)$ & & & & $0.52^{\mathrm{a}}$ \\
\hline Personality disorders, $n$ (\%) & $20(20.0)$ & $12(24.0)$ & $8(16.0)$ & $0.54(1)$ & & & 0.46 \\
\hline Any current anxiety diagnosis, $n$ (\%) & $11(11.0)$ & $4(8.0)$ & $7(14.0)$ & & & & $0.52^{\mathrm{a}}$ \\
\hline
\end{tabular}




\begin{tabular}{|c|c|c|c|c|c|}
\hline & B $(95 \% \mathrm{Cl})$ & $F$ & d.f. & $r^{\mathrm{a}}$ & $P$ \\
\hline \multicolumn{6}{|l|}{ Primary analysis } \\
\hline \multicolumn{6}{|c|}{ Hamilton Rating Scale for Depression } \\
\hline Intercept & 21.39 (20.34 to 22.44 ) & 1630.67 & 98.26 & 0.97 & $<0.001$ \\
\hline Time & $-1.56(-1.67$ to -1.46$)$ & 885.78 & 87.95 & 0.95 & $<0.001$ \\
\hline Time squared & $0.027(0.025$ to 0.029$)$ & 797.22 & 83.20 & 0.95 & $<0.001$ \\
\hline Condition & $-0.72(-2.82$ to 1.38$)$ & 0.46 & 98.26 & 0.07 & 0.499 \\
\hline Time $\times$ condition & $-0.31(-0.51$ to -0.10$)$ & 8.56 & 87.95 & 0.30 & 0.004 \\
\hline Time squared $\times$ condition & 0.005 (0.002 to 0.009$)$ & 7.83 & 83.20 & 0.29 & 0.006 \\
\hline \multicolumn{6}{|l|}{ Beck Depression Inventory } \\
\hline Intercept & 27.87 (26.64 to 29.10$)$ & 2028.61 & 98.17 & 0.98 & $<0.001$ \\
\hline Time & $-2.03(-2.16$ to -1.91$)$ & 1000.78 & 91.56 & 0.96 & $<0.001$ \\
\hline Time squared & 0.034 (0.032 to 0.037$)$ & 907.91 & 88.02 & 0.95 & $<0.001$ \\
\hline Condition & $-1.95(-4.40$ to 0.50$)$ & 2.48 & 98.17 & 0.16 & 0.118 \\
\hline Time $\times$ condition & $-0.32(-0.57$ to 0.06$)$ & 6.31 & 91.56 & 0.25 & 0.014 \\
\hline Time squared $\times$ condition & 0.005 (0.0005 to 0.01$)$ & 4.90 & 88.02 & 0.23 & 0.029 \\
\hline \multicolumn{6}{|l|}{ Sensitivity analyses } \\
\hline \multicolumn{6}{|c|}{ Hamilton Rating Scale for Depression } \\
\hline Intercept & 21.46 (20.41 to 22.52 ) & 1620.34 & 98.47 & 0.97 & $<0.001$ \\
\hline Time & $-1.56(-1.66$ to -1.46$)$ & 926.95 & 92.05 & 0.95 & $<0.001$ \\
\hline Time squared & $0.027(0.025$ to 0.028$)$ & 843.77 & 89.70 & 0.95 & $<0.001$ \\
\hline Condition & $-0.72(-2.83$ to 1.40$)$ & 0.45 & 98.47 & 0.07 & 0.503 \\
\hline Time $\times$ condition & $-0.29(-0.49$ to -0.08$)$ & 7.76 & 92.05 & 0.28 & 0.006 \\
\hline Time squared $x$ condition & 0.005 (0.001 to 0.008$)$ & 6.97 & 89.70 & 0.27 & 0.010 \\
\hline \multicolumn{6}{|l|}{ Beck Depression Inventory } \\
\hline Intercept & 27.67 (26.45 to 28.90$)$ & 2012.54 & 98.21 & 0.98 & $<0.001$ \\
\hline Time & $-2.03(-2.15$ to 1.90$)$ & 990.07 & 90.91 & 0.96 & $<0.001$ \\
\hline Time squared & 0.034 (0.032 to 0.037$)$ & 897.95 & 87.16 & 0.95 & $<0.001$ \\
\hline Condition & $-1.80(-4.24$ to 0.66$)$ & 2.11 & 98.21 & 0.15 & 0.150 \\
\hline Time $\times$ condition & $-0.31(-0.67$ to 0.06$)$ & 5.88 & 90.91 & 0.25 & 0.017 \\
\hline Time squared $\times$ condition & 0.005 (0.000 to 0.009$)$ & 4.31 & 87.16 & 0.22 & 0.041 \\
\hline
\end{tabular}

\begin{tabular}{|c|c|c|c|c|c|}
\hline Moderation & $B(95 \% \mathrm{Cl})$ & $F$ & d.f. & $r^{a}$ & $P$ \\
\hline \multicolumn{6}{|l|}{ Hamilton Rating Scale for Depression } \\
\hline Intercept & 21.43 (20.39 to 22.47 ) & 1679.15 & 85.84 & 0.98 & $<0.001$ \\
\hline Time & $-1.57(-1.69$ to -1.44$)$ & 633.26 & 70.76 & 0.95 & $<0.001$ \\
\hline Time squared & $0.027(0.025$ to 0.029$)$ & 614.71 & 73.49 & 0.95 & $<0.001$ \\
\hline Condition & $-0.16(-2.24$ to 1.92$)$ & 0.02 & 85.74 & 0.02 & 0.88 \\
\hline Baseline severity & 4.61 (3.53 to 5.69$)$ & 72.02 & 87.56 & 0.67 & $<0.001$ \\
\hline Time $\times$ condition & $-0.35(-0.60$ to -0.10$)$ & 7.86 & 70.40 & 0.32 & 0.007 \\
\hline Time squared $\times$ condition & $0.006(0.002$ to 0.010$)$ & 7.75 & 73.14 & 0.31 & 0.007 \\
\hline Time $\times$ baseline severity & $-0.30(-0.43$ to -0.17$)$ & 21.20 & 74.27 & 0.44 & $<0.001$ \\
\hline Time squared $\times$ baseline severity & 0.005 (0.003 to 0.007$)$ & 17.92 & 75.91 & 0.44 & $<0.001$ \\
\hline Condition $\times$ baseline severity & $-2.03(-3.01$ to -1.05$)$ & 16.92 & 92.14 & 0.39 & $<0.001$ \\
\hline \multicolumn{6}{|l|}{ Beck Depression Inventory } \\
\hline Intercept & 26.33 (24.56 to 28.11$)$ & 867.20 & 95.97 & 0.95 & $<0.001$ \\
\hline Time & $-1.88(-2.07$ to -1.68$)$ & 360.04 & 93.79 & 0.89 & $<0.001$ \\
\hline Time squared & $0.032(0.029$ to 0.035$)$ & 361.43 & 92.37 & 0.89 & $<0.001$ \\
\hline Condition & $-8.62(-12.17$ to -5.07$)$ & 23.23 & 95.90 & 0.44 & $<0.001$ \\
\hline Baseline Severity & $3.36(1.52$ to 5.20$)$ & 13.14 & 96.93 & 0.35 & $<0.001$ \\
\hline Time $\times$ condition & $0.36(-0.04$ to 0.75$)$ & 3.26 & 93.47 & 0.18 & 0.074 \\
\hline Time squared $\times$ condition & $-0.006(-0.012$ to 0.008$)$ & 3.02 & 92.07 & 0.18 & 0.086 \\
\hline Time $\times$ baseline severity & $-0.17(-0.37$ to 0.04$)$ & 2.65 & 97.28 & 0.16 & 0.107 \\
\hline Time squared $\times$ baseline severity & $0.003(-0.001$ to 0.006$)$ & 2.23 & 95.03 & 0.15 & 0.139 \\
\hline Condition $\times$ baseline severity & $-2.20(-3.41$ to -1.00$)$ & 13.25 & 92.85 & 0.35 & $<0.001$ \\
\hline
\end{tabular}

participants; and for the TAU group, 12 out of 43 (27.9\%) and 20 out of $43(46.5 \%)$. Behavioural activation differed significantly from TAU in remission rates $\left(\chi_{(1, n=87)}^{2}=12.60, \quad P<0.001\right.$, $\mathrm{OR}=4.99, \quad 95 \%$ CI 2.01-12.44); and in response rates $\left(\chi_{(1, n=87)}^{2}=17.69, \quad P<0.001, \quad\right.$ OR $=8.97,95 \%$ CI $\left.2.96-27.14\right)$. From those that remitted at 13 weeks, 10 out of $36(27.8 \%$ behavioural activation group) and 12 out of $20(60.0 \%$ TAU group) relapsed at 49 weeks. This difference was also significant $\left(\chi_{(1, n=56)}^{2}=5.60, P=0.018, \mathrm{OR}=3.90,95 \%\right.$ CI $\left.1.23-12.37\right)$. At 49 weeks, 3 out of $44(6.8 \%)$ in the behavioural activation group and 11 out of $43(25.6 \%)$ in the TAU group used antidepressant medication $\left(\chi_{(1, n=87)}^{2}=5.67, P=0.017\right)$. 

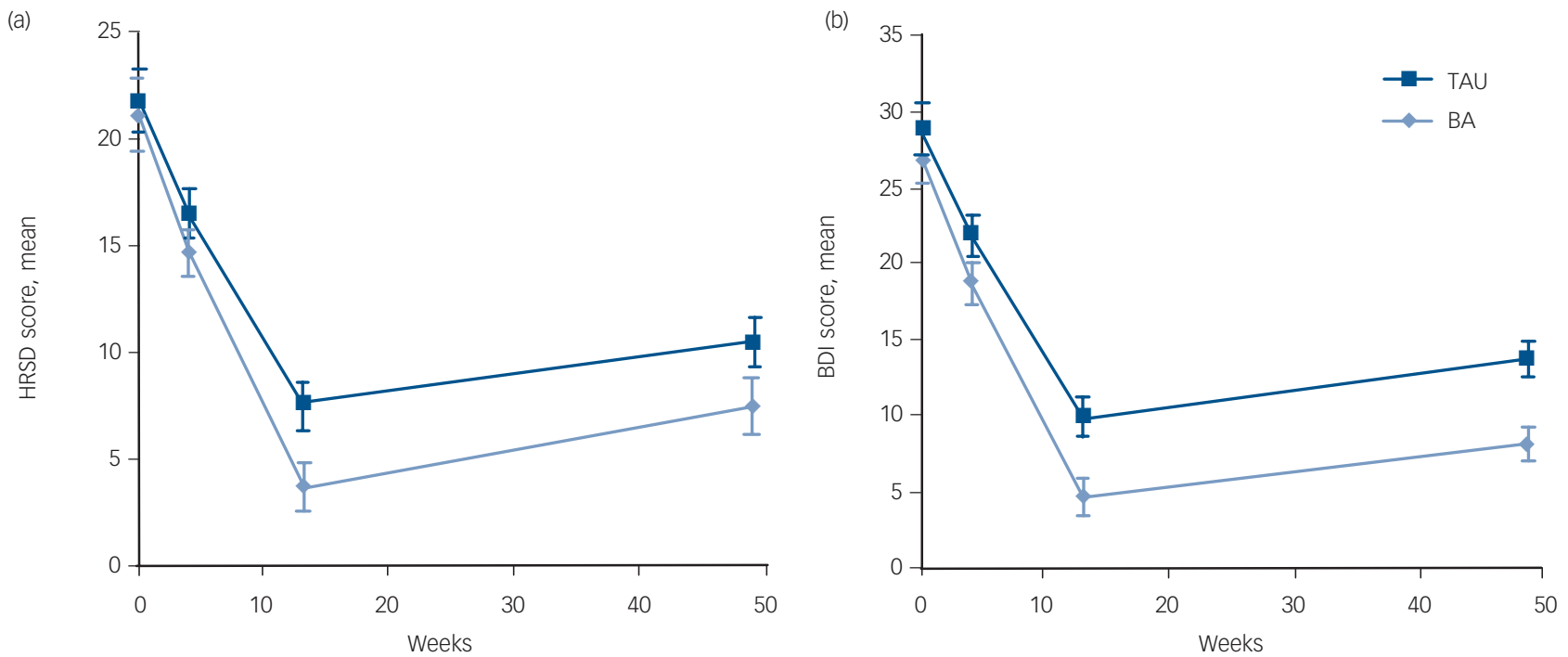

Fig. 2 Mixed regression-based estimated means and 95\% Cl of Hamilton Rating Scale for Depression (HRSD) and Beck Depression Inventory (BDI) at baseline, 4, 13 and 49 weeks for behavioural activation (BA) and treatment as usual (TAU).
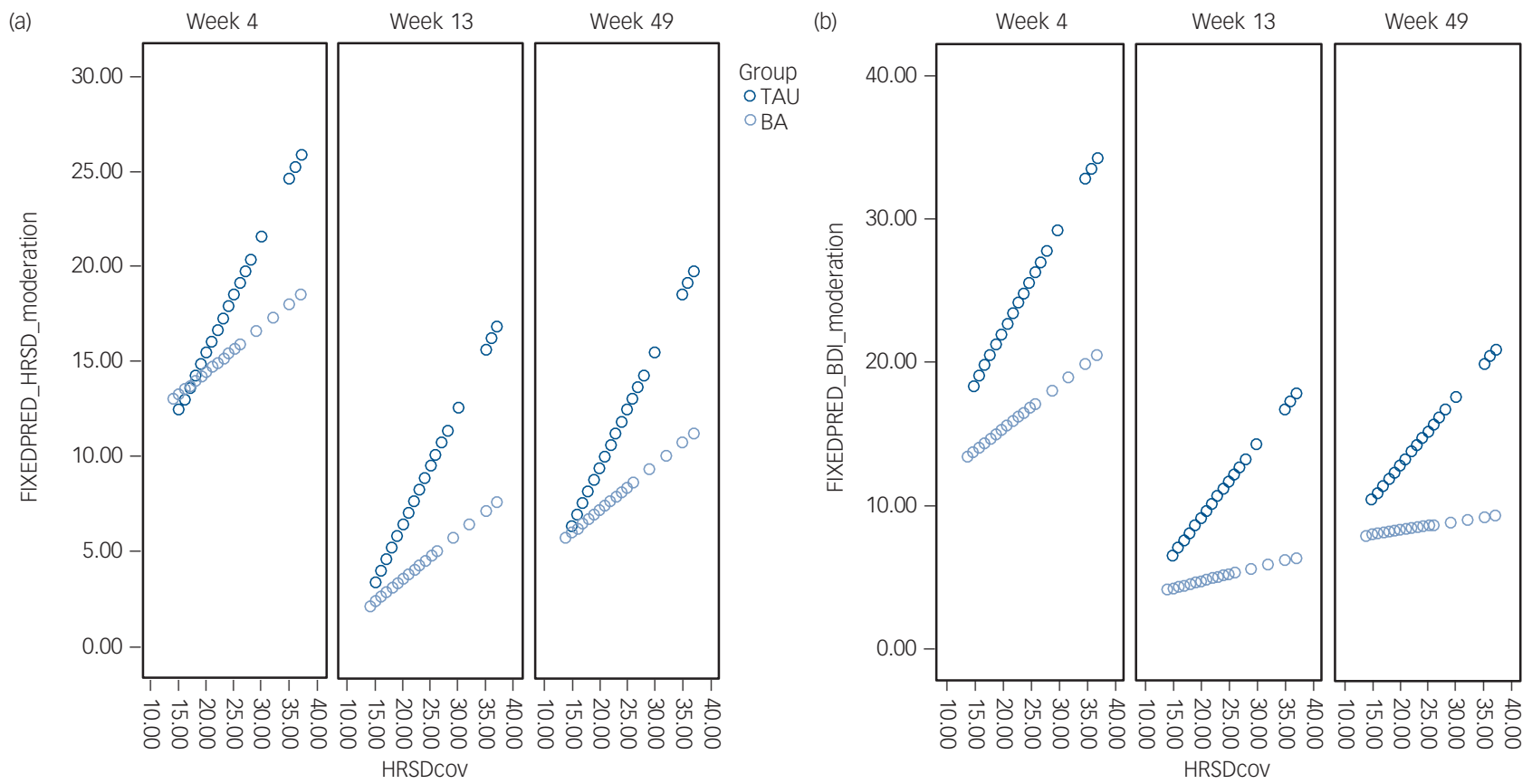

Fig. 3 Moderation of condition effects by baseline severity (Hamilton Rating Scale for Depression (HRSD) score at baseline) at 4-, 13- and 49-week assessments on the (a) HRSD and (b) Beck Depression Inventory (BDI): fixed predicted scores from mixed regression analyses.

Individuals in the behavioural activation (BA) group had on average lower HRSD and BDI scores, but the higher the baseline severity, the stronger the difference between behavioural activation and treatment as usual (TAU). HRSDcov, HRSD score at baseline, represeting baseline severity.

\begin{tabular}{|c|c|c|c|c|c|c|c|c|c|c|}
\hline & \multicolumn{2}{|c|}{ Baseline } & \multicolumn{4}{|c|}{ Baseline to week 13} & \multicolumn{4}{|c|}{ Baseline to week 49} \\
\hline & \multirow{2}{*}{$\begin{array}{c}\text { HRSD } \\
\text { Baseline s.d. }\end{array}$} & \multirow{2}{*}{$\frac{\text { BDI }}{\text { Baseline s.d. }}$} & \multicolumn{2}{|c|}{ HRSD } & \multicolumn{2}{|c|}{$\mathrm{BDI}$} & \multicolumn{2}{|c|}{ HRSD } & \multicolumn{2}{|c|}{$\mathrm{BDI}$} \\
\hline & & & Mean & $d$ & Mean & $d$ & Mean & $d$ & Mean & $d$ \\
\hline Behavioural activation & 5.39 & 6.34 & -17.31 & 3.24 & -22.28 & 3.51 & -13.58 & 2.54 & -18.79 & 2.96 \\
\hline Treatment as usual & 5.39 & 6.34 & -14.22 & 2.66 & -18.94 & 2.99 & -11.24 & 2.11 & -15.12 & 2.39 \\
\hline
\end{tabular}




\section{Discussion}

The findings of this study indicate that behavioural activation is superior to antidepressant medication in terms of effectiveness, especially in those with severe depression, and the prevention of relapse. The lower drop-out rate in the behavioural activation group suggests that behavioural activation was better tolerated than medication. As it was also more effective, behavioural activation might offer a viable alternative to medication, especially for those who prefer non-pharmacological treatment. The results replicate and extend findings of three earlier trials ${ }^{12,13,16}$ and emphasise the value of simple behavioural strategies in the treatment of depression.

Until now, behavioural activation has been tested by the developers ${ }^{12,13}$ and by only one independent study. ${ }^{16}$ In our study, behavioural activation therapists were not trained and supervised by behavioural activation experts but by a non-expert using the published protocol, which is encouraging regarding the feasibility of its dissemination around the globe. Behavioural activation interventions are relatively simple and easy to understand for individuals with depression, and do not require difficult or complex skills on the part of participants or therapists. ${ }^{25}$ It could therefore be considered as a first-choice treatment, with potentially good cost-effectiveness.

In Dimidjian et al's study ${ }^{13}$ behavioural activation was conducted over 24 sessions over a 4-month period, as opposed to the 16 sessions in our study. Remission and response rates were higher in our study (remission and response rates in the behavioural activation group of $91.1 \%$, and $97.8 \%$ respectively) compared with the rates in the high severity group in the Dimidjian et al study ${ }^{13}$ (remission and response rates in the behavioural activation group of $52-56 \%$ and $60-76 \%$ respectively). Reasons for the striking effectiveness of behavioural activation in our study might be that behavioural activation fits particularly well with the Iranian culture. Although avoidance and rumination are typical responses observed in all people with depression, they might be even more typical in the Iranian context. The behavioural activation therapists had the impression that behavioural activation strategies that tackle rumination led to a strong reduction in depressive symptoms. In Iran, psychological treatment seems more acceptable than pharmacological treatment, because seeing a psychologist is more accepted (as the reason is that one merely has 'life problems'), whereas pharmacological treatment is associated with having 'mental disease', which has a heavy cultural stigma, especially for men.

Several studies have reported substantial relapse after discontinuation of antidepressant medication. ${ }^{10,26,27}$ From a behavioural activation perspective, a likely reason is that people who are treated with antidepressants and then relapse did not change their coping skills. Patterns of avoidance, environmental punishment, lack of reinforcement, and depressive rumination might still exist, although their effect on mood might have been temporarily reduced through the use of antidepressants. In contrast, behavioural activation equips individuals with healthy behavioural skills and thus the probability of relapse is reduced.

The difference in effectiveness between behavioural activation and antidepressant medication also points to differential pathways of change. Behavioural activation and antidepressants are obviously likely to work through different mechanisms, but this study provides further evidence that activation is indeed a crucial element that leads to improvement in psychotherapy for depression. The original study by Jacobson et al ${ }^{12}$ was set up to identify the active components of CBT, and found that behavioural activation was at least as effective as the complete package of CBT.
Dimidjian et al $^{13}$ replicated this finding, and found behavioural activation to be even more effective than CBT in severe depression. Although behavioural activation in our study is not compared with CBT, the findings underline that simple activation strategies in psychotherapy are essential. It should be noted, however, that this study provides merely indirect evidence for a causal link between activation and recovery in depression and that experimental studies are needed to reveal the working mechanisms of behavioural activation.

\section{Limitations}

A number of limitations should be mentioned. First, the fact that therapists were not trained by behavioural activation experts might have led to suboptimal application of the behavioural activation techniques. Furthermore, participants had to pay for antidepressant medication after 3 months, which might have contributed to discontinuation. However, these two limitations can also be considered as strengths from the point of view of effectiveness research, which addresses how the two approaches compare in routine practice settings. A third limitation is the high proportion of participants recruited by advertising and word of mouth. It is unclear to what degree the sample is different from regular clinical samples in Iran. Fourth, the rate of attrition in the antidepressant medication group was relatively high and this could have influenced the results. Fifth, we did not change the prescribed drug, sertraline, for the patients in the case of non-response. However, this was the same for behavioural activation. Sixth, independent assessments of treatment integrity and reliability checks of the HRSD interviews were not done because of financial constraints. Seventh, the sample may have been biased in that the study especially attracted participants preferring psychological treatment, which is not widely available in Iran. Eighth, the absence of a non-treatment or placebo condition is another limitation. Ninth, we did not control for the amount of attention participants received from their therapists. The weaker effects of antidepressant medication might be partially explained by this. Finally, the majority of our sample was female, and although this reflects clinical practice in Iran, further studies are needed into non-Western male samples.

\section{Future research}

Our finding that behavioural activation was superior to antidepressant medication, and even more so in the more severely depressed subgroup, should be replicated by other groups and in other settings. Subsequent trials in which the effectiveness and cost-effectiveness of behavioural activation, antidepressant medication and other psychological treatments, such as cognitive therapy, are investigated are indicated.

Another issue pertains to mechanisms of change that are accountable for the superior effects of behavioural activation, as mentioned above. Understanding the (differential) causal pathways that lead to recovery with behavioural activation and antidepressants might illuminate important insights that help to improve treatment strategies in depression, and future research is warranted here.

\section{Implications of this study}

The findings of our study suggest that behavioural activation is a simple and effective intervention for depression that can be easily disseminated to routine practice settings, similar to what has been demonstrated in Western countries. ${ }^{16}$ The fact that the behavioural activation was delivered effectively by therapists with a minimum of training and supervision is very encouraging, taken together 
with the superior effects in the subgroup of participants with more severe depression, it speaks for its timely dissemination to other routine practice settings as well. Moreover, behavioural activation might be a very cost-effective intervention. Although our study did not include a formal economic evaluation, we calculated that the total direct treatment costs for behavioural activation participants amounted to $€ 128$ per patient, whereas the total direct treatment costs for those participants treated with antidepressants were $€ 198$ per patient on average, depending on the number of followup consultations. It has even been suggested that behavioural activation can be provided by healthcare professionals who had no previous experience with providing psychotherapy, ${ }^{16}$ which further increases possibilities for its implementation.

Latif Moradveisi, MSc, Department of Clinical Psychological Science, Faculty of Psychology and Neuroscience, Maastricht University, The Netherlands, and Department of Psychiatry, Faculty of Medicine, Kurdistan University of Medical Science, Sanandaj, Iran; Marcus J. H. Huibers, PhD, Department of Clinical Psychology, VU University, Amsterdam, The Netherlands, Fritz Renner, MSc, Department of Clinical Psychological Science, Faculty of Psychology and Neuroscience, Maastricht University, The Netherlands; Modabber Arasteh, MD, Department of Psychiatry, Faculty of Medicine, Kurdistan University of Medical Science, Sanandaj, Iran; Arnoud Arntz, PhD, Department of Clinical Psychological Science, Faculty of Psychology and Neuroscience, Maastricht University and Netherlands Institute for Advanced Study in the Humanities and Social Sciences, Wassenaar, The Netherlands

Correspondence: Arnoud Arntz, Department of Clinical Psychological Science, Faculty of Psychology and Neuroscience, Maastricht University,

PO Box 616, 6200 MD Maastricht, The Netherlands. Email: Arnoud.Arntz@ maastrichtuniversity.nl

First received 20 Apr 2012, final revision 17 Nov 2012, accepted 10 Dec 2012

\section{Funding}

Maastricht University and Kurdistan University of Medical Sciences provided the financia support of this study.

\section{Acknowledgements}

The authors gratefully acknowledge their colleagues for their contributions to this study: Azad Maroufi, Farzin Rezaei and Marzia Moghadam (along with M.A.) for providing antidepressant medication therapy; Farhad Asghari and Reza Mozhdehi for providing behavioural activation (along with L.M.); Yahya Yarahmadi for conducting baseline diagnostic interviews; Farshid Rastgari and Ebrahim Hoseini for conducting the HRSD interviews; Ladan Khaledi for coordinating assessments and administering the BDI. Pedram Andalibi provided sertraline in his drugstore free of charge. Jafar Mirzaei and Mohsen Ghadermarzi coordinated the preparation of the treatment setting and Fardin Gharibi supported and coordinated the study. Ahmad Sohrabi instructed the authors how to use the Iranian SCID-I.

\section{References}

1 American Psychiatric Association. Diagnostic and Statistical Manual of Mental Disorders (4th edn) (DSM-IV). APA, 1994.

2 Blacker D. Maintenance treatment of major depression: a review of the literature. Harv Rev Psychiatry 1996; 4: 19.

3 Fein S, Paz V, Rao N, Lagrassa J. The combination of lithium carbonate and an MAO in refractory depression. Am J Psychiatry 1988; 145: 249-50.

4 Hirschfeld RMA, Schatzberg AF. Long-term management of depression. Am J Medicine 1994; 97: 338
5 Schatzberg AF. Treatment of severe depression with the selective serotonin reuptake inhibitors. Depress Anxiety 1996; 4: 182-9.

6 American Psychiatric Association. Practice guidelines for the treatment of patients with major depressive disorder (revision). Am J Psychiatry 2000; 157: 1-45.

7 Olfson M, Marcus SC, Druss B, Pincus HA. National trends in the use of outpatient psychotherapy. Am J Psychiatry 2002; 159: 1914-20.

8 Dobson KS, Hollon SD, Dimidjian S, Schmaling KB, Kohlenberg RJ, Gallop RJ, et al. Randomized trial of behavioral activation, cognitive therapy, and antidepressant medication in the prevention of relapse and recurrence in major depression. J Consult Clin Psychol 2008; 76: 468-77.

9 Hollon SD, Shelton RC. Treatment guidelines for major depressive disorder. Behav Ther 2001; 32: 235-58.

10 Gloaguen V, Cottraux J, Cucherat M, Blackburn IM. A meta-analysis of the effects of cognitive therapy in depressed patients. J Affect Disord 1998; 49 : 59-61.

11 Beck AT, Rush AJ, Shaw BF, Emery G. Cognitive Therapy of Depression. Guilford Press, 1979

12 Jacobson NS, Dobson KS, Truax PA, Addis ME, Koerner K, Gollan JK, et al. A component analysis of cognitive-behavioral treatment for depression. J Consult Clin Psychol 1996; 64: 295-304.

13 Dimidjian S, Hollon SD, Dobson KS, Schmaling KB, Kohlenberg RJ, Addis ME, et al. Randomized trial of behavioral activation, cognitive therapy, antidepressant medication in the acute treatment of adults with major depression. J Consult Clin Psychol 2006; 74: 658-70.

14 Cuijpers $P$, Van Straten A, Warmerdam L. Behavioral activation treatment of depression: a meta-analysis. Clin Psychol Rev 2007; 27: 318-26.

15 Ekers D, Richards D, Gilbody S. A meta-analysis of randomized trials of behavioral treatment of depression. Psychol Med 2008; 38: 611-23.

16 Ekers D, Richards D, McMillan D, Bland JM, Gilbody S. Behavioral activation delivered by the non-specialist: phase II randomised controlled trial. $\mathrm{Br}$ J Psychiatry 2011; 198: 66-72.

17 Martell CR, Dimidjian S, Herman-Dunn R. Behavioral Activation for Depression: A Clinician's Guide. The Guilford Press, 2010

18 Martell CR, Addis ME, Jacobson NS. Depression in Context: Strategies for Guided Action. Norton Press, 2001

19 American Psychiatric Association. Diagnostic and Statistical Manual of Mental Disorders (4th edn, text revision) (DSM-IV-TR). APA, 2000.

20 First MB, Williams, JB, Spitzer RL, Gibbon M. Structured Clinical Interview for DSM-IV-TR Axis I Disorders, Clinical Trials version (SCID-CT). Biometrics Research, New York State Psychiatric Institute, 2007.

21 Beck AT, Steer RA, Brown GK. Manual for the BDI-II. Psychological Corporation, 1996.

22 Hamilton M. A rating scale for depression. J Neurol Neurosur Psychiatry 1960; 23: 56-61.

23 Sadock BJ, Sadock VA. Kaplan and Sadock's Synopsis of Psychiatry: Behavioral Sciences/Clinical Psychiatry (10th edn). Lippincott Williams \& Wilkins, 2007

24 First MB, Spitzer RL, Gibbons M, Williams JB, Benjamin L. User's Guide for the Structured Clinical Interview for DSMIV Axis II Personality Disorders (SCID-II). New York State Psychiatric Institute, Biometrics Research Department, 1996.

25 Lejuez CW, Hopko DR, LePage JP, Hopko SD, MCNeil DW. A brief behavioral activation treatment for depression. Cogn Behav Pract 2001; 8: 164-75.

26 Hollon SD, DeRubeis RJ, Shelton RC, Amsterdam JD, Salomon RM, O'Reardon $J P$, et al. Prevention of relapse following cognitive therapy vs. medications in moderate to severe depression. Arch Gen Psychiatry 2005; 62: 417-22.

27 Evans MD, Hollon SD, DeRubeis RJ, Piasecki JM, Grove WM, Garvey MJ, et al. Differential relapse following cognitive therapy and pharmacotherapy for depression. Arch Gen Psychiatry 1992; 49: 802-8. 